\title{
BARRIERS AND FACILITATORS OF AREA-WIDE MANAGEMENT INCLUDING STERILE INSECT TECHNIQUE APPLICATION: THE EXAMPLE OF QUEENSLAND FRUIT FLY
}

\author{
A. MANKAD ${ }^{1}$, B. LOECHEL ${ }^{1}$ AND P. F. MEASHAM ${ }^{2}$ \\ ${ }^{1}$ CSIRO Land \& Water, GPO Box 2583, Brisbane QLD 4001, Australia; \\ aditi.mankad@csiro.au; barton.loechel@csiro.au \\ ${ }^{2}$ Hort Innovation, 527 Gregory Terrace, Fortitude Valley QLD 4006, Australia; \\ penny.measham@horticulture.com.au
}

\begin{abstract}
SUMMARY
The area-wide management (AWM) of highly mobile insect pests such as tephritid fruit flies requires an integrated understanding of technical, social and institutional processes that drive a coordinated approach within a defined area. Furthermore, the success of an AWM programme is dependent upon the coordinated efforts of key stakeholders within the designated area (e.g. growers, community members). Yet, public views regarding AWM may not reflect those views held by scientists or stakeholders. Public considerations for acceptance are likely varied and multidimensional. A series of qualitative (phases 1-2) and quantitative (phase 3) studies examined stakeholder and community attitudes towards AWM to manage Queensland fruit fly Bactrocera tryoni (Froggatt) (Tephritidae) and the novel use of the Sterile Insect Technique (SIT) as a possible component of AWM. Research was conducted over three regions of varying pest prevalence, ranging from zero to endemic; participants included growers, extension officers, industry and government representatives, and members of the general public. Participants in this research were asked to consider potential barriers and facilitators to the widespread uptake of AWM integrating the SIT, including any relevant institutional-level factors. Combined data revealed potential social barriers to AWM and SIT uptake. Most notably, there were perceptions of low efficacy in successfully coordinating key social groups for the purposes of an AW-IPM approach, and a concern for the possibility of 'free-riders' within an areawide system. On the other hand, innovation complexity and observability of outcomes were important factors contributing to acceptance of AWM. Importantly, all participants were influenced by the attitudes and behaviours of important others. Participants also identified key facilitators that could assist in the uptake of AWM using the SIT. These facilitators include the importance of trustworthy information sources and harnessing the persuasive influence of community champions and central packing houses on commercial growers. Overall, there was high stated acceptance for the SIT, both on-farm and in towns, as long as SIT application was found to be economically feasible at individual farm or household level and the community was adequately consulted.
\end{abstract}

669

J. Hendrichs, R. Pereira and M. J. B. Vreysen (eds.), Area-Wide Integrated Pest Management: Development and Field Application, pp. 669-692. CRC Press, Boca Raton, Florida, USA.

(C) 2021 IAEA 
Key Words: Bactrocera tryoni, Tephritidae, social science, biosecurity, integrated pest management, public attitudes, behavioural science, stakeholders, coordination, social barriers, technology uptake, Australia

\section{INTRODUCTION}

The area-wide management (AWM) of mobile insect pests such as tephritid fruit flies requires a multidimensional integrated understanding of technical, social and institutional processes that drive a coordinated approach within a defined area. Such management of agricultural pests is dependent upon the success and efficacy of the technical treatments associated with control, as well as the social environment within which the management takes place. Indeed, the central tenet of AWM is coordination amongst all relevant stakeholders. This inherently implies an element of social interaction to achieve effective pest management over a region. In the AWM context, people with varying interests must come together for a common goal. Thus, there must be an understanding of the social and institutional mechanisms in place to enable the implementation of coordinated activities.

This chapter focuses on a series of qualitative (phase 1-2) and quantitative (phase 3) studies examining stakeholder and community attitudes towards AWM to control Queensland fruit fly, Bactrocera tryoni (Froggatt), for the eventual integration of the Sterile Insect Technique (SIT) as a component of AWM. Through phases 1 and 2 of this social science research, contextual barriers and facilitators of AWM - as perceived by farmers and members of the general public - were identified. Predictions could also be made with respect to underlying attitudinal drivers of acceptance of AWM and the integration of the SIT, and intentions to participate in an AWM approach, at the completion of phase 3. While AWM can take place without the integration of the SIT, the focus of this project was AWM for eventual SIT implementation. Therefore, the results are discussed in this context.

\subsection{Queensland Fruit Fly: A Significant Horticultural Pest}

Prior to European settlement of Australia, the Queensland fruit fly was considered endemic to the tropical and subtropical rainforests of north-eastern Australia (Meats 1981; Reynolds and Orchard 2015). It has subsequently spread from this habitat and is now considered endemic in most of east-coast mainland Australia, including temperate areas, except where under regulatory control (Dominiak and Daniels 2012). In Queensland, B. tryoni occurs in high numbers year-round with 3-4 generations per year in the southern areas (Meats 1981). Meats (1981) believed that populations in temperate regions were transient, due to populations immigrating in each season but not establishing year-round. However, there is now clear evidence that the fly is permanently established in temperate eastern Australia (Dominiak and Daniels 2012; Reynolds and Orchard 2015; Agriculture Victoria 2017; Dominiak and Mapson 2017).

Many fruit industries in Australia are looking to increase exports in the future, which come with a concomitant need to be able to meet protocol requirements of trading partners, especially in crop monitoring and compliance. Achieving pest-free status and increased market access is important, but equally important is maintaining 
current trade through effective control strategies. With a very large host range, more than three-quarters of Australian fruit and vegetable exports are susceptible to Queensland fruit fly (Clarke et al. 2014). Not surprisingly, therefore, this pest causes significant difficulties for producing clean fruit and developing market access. On average, the annual value of fruit fly susceptible production in Australia is over AUD 5000 million (between 2006 and 2009) (Abdalla et al. 2012). Without fruit fly control, production losses due to B. tryoni have been estimated at $80-100 \%$ (Sutherst et al. 2000 ) and even with available management efforts, estimates of production losses in endemic areas range from 0.5 to $3 \%$ annually depending on crop type (Abdalla et al. 2012). Queensland fruit fly is also considered a significant barrier to market access for Australian horticultural products (Ekman 2015). Pest-free status provides producers within such declared regions a significant advantage; Tasmania, for example, exported nearly $50 \%$ of its total cherry yield to China, compared to less than $1 \%$ from mainland Australia (CGA 2015). In order to export to either regulated domestic or international markets, producers in B. tryoni endemic areas must comply with specific requirements; protocols generally dictate a Probit 9 end-point phytosanitary treatment, such as cold storage or fumigation with methyl bromide. These treatments assume growers implement a level of pre-harvest and post-harvest control, both incurring costs (Lloyd et al. 2010).

With the loss of regulatory fruit fly area-freedom in some parts of temperate eastern Australia (Agriculture Victoria 2017; Dominiak and Mapson 2017), and the regulatory loss of some chemical options for Queensland fruit fly (Dominiak and Ekman 2013; Florec et al. 2013), new control options for B. tryoni are needed. As AWM is an internationally recognised approach for mobile pests (Vreysen et al. 2007a), this seems a sensible approach to achieving effective control of Queensland fruit fly, while underpinning market access requirements. The application of an AWM approach within any chosen region or area requires the integration of control tactics (Chandler et al. 1999; Suckling et al. 2014), as the use of a single management tool is deemed insufficient to suppress effectively mobile pests (Vargas et al. 2010). AWM should also be seen as a long-term undertaking, with long-term solutions (Hendrichs et al. 2007; Vander Meer et al. 2007; Vreysen et al. 2007a; Yu and Leung 2011; Ogaugwu 2014). B. tryoni control and surveillance tools currently available in Australia and being used in-field include:

- Monitoring - assessing pest population trends through male pheromone traps

- Bait spraying - a protein (usually yeast-based) and toxicant, targeting female flies that are attracted to the food bait

- Male annihilation technique (MAT) - high density placement of a male attractant lure (cue-lure) and toxicant, targeting male flies (to be effective, MAT needs to "attract and kill" most males in a population)

- Orchard sanitation - the systematic removal of fallen and infested fruit

- Cover sprays - for some crops where registration allows, when pest pressure is high.

The general consensus is that bait-spraying and MAT are complementary measures, while sanitation is an essential key component of many fruit fly pest management approaches (Dominiak et al. 2015; Stringer et al. 2017). The release of sterile male flies embedded into a well-managed AWM programme that integrates the 
above control measures can be highly successful as shown in other parts of the world (Fisher et al. 1985; Fisher 1996; Enkerlin et al. 2005; Pereira et al. 2013), but has yet to be implemented on a large-scale in Australia.

\subsection{Methodological Approaches}

The methodological approaches chosen in this series of studies were complementary, where each phase built upon the previous phase:

Phase 1 comprised one-on-one interviews with fruit growers and industry representatives. Participants were asked about their attitudes towards Queensland fruit fly as a personal threat, as well as a threat to the fruit-growing industry.

Phase 2 was a series of focus groups with a wider range of "stakeholder" groups: fruit growers and packers, government and industry representatives, extension officers, and the general public. The content of the focus groups was an extension of the interview phase and provided more in-depth examination of the issues raised during interviews. Participants explored possible motivations for participating in AWM activities, as well as offering potential scenarios for rules, regulations and AWM and SIT funding. General public perspectives were also incorporated at phase 2.

Finally, phase 3 involved a broad-scale telephone survey of fruit growers and members of the general public. The purpose of the survey was to examine key factors, identified through the qualitative work, on a larger and more measurable scale. This was to determine the relative importance of different social and institutional factors on acceptance and intention around AWM and SIT implementation. However, the issues discussed in this chapter are predominantly derived from phases 1 and 2 of our research survey programme, which are rich in contextual data. It is also important to note that the scale of our social research was an examination of manageable farmerfarmer or neighbour-neighbour coordination options. Given the extensive length and breadth of Australian horticultural landscapes, our phase 1 results indicated that it was not feasible to consider interpersonal social factors of AWM collaboration beyond that scale.

This process of multi-method (quantitative and qualitative) data collection and analysis allowed an exploratory approach to identifying relevant social issues, without imposing any researcher bias on possible barriers or enablers that might be considered theoretically important. Therefore, a strength of this research is that the social factors covered within this chapter are participant-driven and derived from farmer and public perspectives. The associated theoretical underpinnings were chosen based on the emergent data, rather than preselecting a theoretical framework to analyse the social landscape.

\subsection{Regional Descriptions}

There were three study regions across south-eastern Australia, each of which is a dominant horticultural production area (see Fig. 1); however, the regions vary with respect to their 'fruit fly status'. 
Region A is now recognised as an endemic area for Queensland fruit fly. Although some parts of region A have pockets of land where levels of Queensland fruit fly are undetectable, the region as a whole is not considered pest-free. Region B is a regulated region with traditionally low Queensland fruit fly prevalence, now with a suspended pest-free status. This is due to many recent outbreaks and signs of early invasion in many parts of the region.

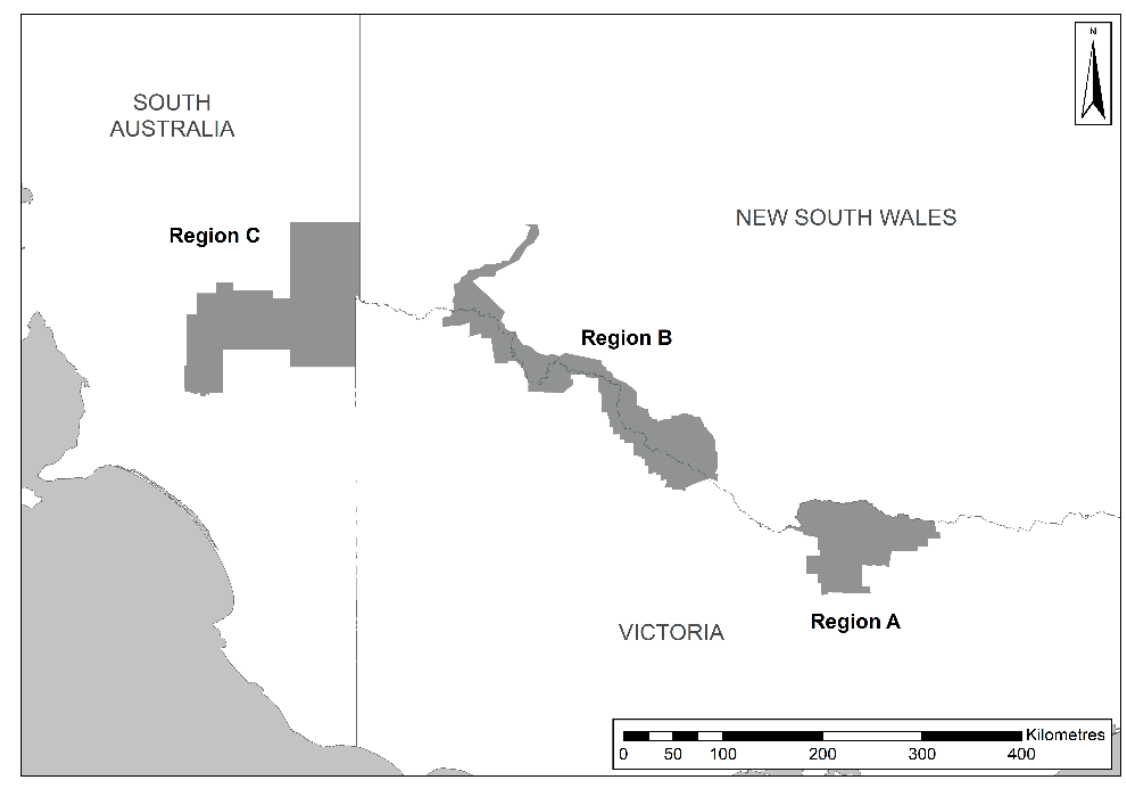

Figure 1. Map of the three study regions targeted for social science research across southeastern Australia: Regions A and B, both spanning New South Wales and Victoria; and Region C, on the South Australian border (source Andy Hulthen, CSIRO).

Finally, region C is a recognised and regulated Queensland fruit fly free area in which any incursions are immediately eradicated by the relevant government authorities (as per International Standard for Phytosanitary Measures (ISPM) Number 26 (FAO 2016)). Until only a few years ago all three regions were under regulatory area freedom (Dominiak and Mapson 2017).

Potential institutional issues related to on-the-ground coordination for AWM in these three regions include regulatory boundaries such as local government areas, state borders, and other nationally recognised boundaries. Flies do not recognise or respect these geographic and administrative delineations that can complicate efforts to coordinate and fund AWM initiatives. Rules and regulations within jurisdictions, or a lack of legal authority (Hendrichs et al. 2007), can also frustrate AWM efforts. Differing organizational goals and rationales, such as those for private firms, government agencies and industry bodies, can further complicate coordinated action. Thus, getting the institutional arrangements 'right' appears a crucial element to effective AWM. 


\section{INSTITUTIONAL BARRIERS AND FACILITATORS FOR AWM}

AWM can be viewed as a pest management innovation that is social, organizational and logistical in nature, as much as it is technological. While it incorporates biophysical technologies as part of its integrated pest management approach, it fundamentally requires cooperation between parties across geographic space. These parties can be diverse, ranging from commercial growing enterprises, to hobbyfarmers, local councils, government agencies, and the general public. Contribution and coordination of these diverse groups can also occur at different levels (e.g. local, state, and national industry bodies).

AWM, theoretically, can also be implemented at different scales from activities organised at the local community level to regional and even national implementation programmes (Kruger 2017a, 2017b). Queensland fruit fly is reported to travel only a relatively short distance (compared to other mobile horticultural pests in Australia) in the presence of hosts and other resources (Dominiak 2012). This suggests grower-togrower AWM for pest suppression would be effective at relatively small scales when growers are in close proximity and any adjoining non-commercial land was included in the programme (Barclay et al. 2011). Larger scale AWM initiatives for broader control may include the integration of multiple grower groups. This may include towns and settlements and their peri-urban fringes, and other host areas such as hobbyfarms and nature reserves (Kruger 2016; Vreysen et al. 2007a). This may require government coordination.

Psychosocial factors that influence participation and cooperation, such as attitude, motivation and trust, are clearly important for sustained collective action. However, there are also a range of institutional factors are also important for coordination (Mankad 2016; Mankad et al. 2017). Institutional factors refer to the various forms and levels of rules and rule-sets that guide human behaviour and the arrangements between participants in a social system (Hodgson 2006). Social systems can be viewed as being comprised of structures, including formal institutions (as the effect or manifestation of rules) and participants or agents (individuals and organizations), whose behaviour is influenced by these institutions (Giddens 1984). Thus, institutions are referred to as the 'rules of the game' and counter-posed to agents as the 'players of the game' (North 1990). Rules are, of course, developed and enacted by people. Therefore, there is a dynamic relationship between institutions and actors (or social structures and agents) where rules influence the behaviour of actors, who at the same time are individually or collectively seeking to reshape the rules to meet their own particular goals (Giddens 1984; Hodgson 2007).

Institutions comprise a broad range of rule types, from formal to informal, such as policies, laws, regulations, protocols, guidelines, procedures, standards, conventions and norms. These establish particular ways of behaving and organising. Institutional factors enable a broad range of programmes and initiatives, such as raising awareness and education campaigns; research, development and extension programmes and facilities; pest monitoring networks, exclusion zones and diagnostic facilities.

This research identified a broad range of institutional factors that could potentially facilitate or constrain participation in AWM for Queensland fruit fly control. Typically, most of the constraints or barriers emerging from the research covered in 
this chapter relate to a deficit of those factors cited as important for the facilitation of AWM. Notable institutional facilitators of AWM that were cited included:

- Formal engagement of stakeholders and consistent communication by industry and government in raising awareness and knowledge building about Queensland fruit fly and AWM

- Integration of efforts across stakeholder groups (growers, government, industry and the general public)

- Cooperation within and between AWM groups and at higher levels of regional organization

- Leadership and governance aspects important to galvanising, guiding and sustaining these various levels of collective action

- Rules, regulation, legislation and enforcement required to achieve greater cooperation and compliance for broad pest suppression across multiple host areas and to raise funds to support AWM

- The potential of greater access to premium (typically export) markets and the role that market access 'protocols' play in this.

The following Sections provide some examples from three key areas that were identified as particularly important.

\subsection{Stakeholder Cooperation and Integration}

Cooperation between parties and integration of efforts is of crucial importance for AWM, whether at a local level (between neighbouring growers and other property managers), at a broader community level (between grower groups and a nearby town council/local government authority to suppress urban pest populations), or a wholeregion level (involving multiple stakeholders representing industry, government and the general public to guide a regional approach). Cooperation at a local level is required mainly to ensure as many growers/property managers are controlling the fly as possible. Due to the often-close proximity of properties to one another, as a mobile pest, Queensland fruit fly can readily reinfest a 'managed' farm from an adjoining 'unmanaged' property. Coordinated timing of control operations is essential for areawide suppression of a pest population, especially with regard to SIT application. Optimal conditions for SIT integration typically include an existing high level of sanitation and suppression, or low-level populations (Stringer et al. 2017), and avoiding insecticide spraying at the time of SIT releases to prevent destroying the sterile flies as they enter the environment.

Participants in the qualitative studies indicated a strong awareness of the need for cooperation across growers and other property managers. They often expressed concern that a small number of uncooperative growers (e.g. apathetic or negative), those unable to cooperate (e.g. elderly or absentee owners), or those for whom the benefits may be limited (e.g. host produce is relatively unaffected by fruit fly), could undermine the success of their own efforts. Concern was also expressed by growers regarding cooperation by urban communities. Rural towns and their backyards were generally perceived as the greatest host risk of population build-up in endemic areas, providing a permanent supply of the pest to infect surrounding commercial growing areas (that were generally perceived as having lower levels of infestation). 
Cooperation by the local government authority or 'council' was perceived as important to the success of an AWM initiative. Councils were considered the main route to organising pest management activities in towns. This was confirmed by participant responses from town residents, and members of councils themselves. Coordinated activities generally involved gaining the cooperation of residents (property owners and/or rental tenants) in agreeing to deploying traps and/or the removal of host fruit/trees in their garden. General public participants in this research further indicated a high willingness to cooperate with their local council in undertaking Queensland fruit fly control practices on their property.

Stakeholder cooperation at a broader level was seen as important to guide and support a strategic approach to AWM at the regional level. This included the cooperation and integration of efforts of different fruit industries (citrus, stone fruit, grape, etc.), different industry segments (growers, fruit packing houses, industry associations, crop pest consultants and agricultural chemical suppliers), and different levels of government (federal, state and local). Cooperation was seen as essential to ensure a better integrated approach that provided a planned and strategic direction and consistency of messaging across the various stakeholder groups. This would ensure a common language and knowledge base, and thereby minimise confusion about what to do. A united approach at this higher stakeholder group level was also seen as important to gain more widespread action on the ground, at the grower level and amongst the general public, to minimise the incidence of untreated areas and 'social loafing', also referred to as 'free-riding'.

\subsection{Education, Engagement, Communication}

The importance of education, engagement and communication comprised a prominent theme in the qualitative findings of this study. Interviews and focus group participants strongly indicated that building high regional awareness of the threat of Queensland fruit fly and a strong understanding about its control, including through AWM and SIT integration, would be crucial to their success. For this to occur, education, communication and engagement activities were seen as paramount, especially using methods and forms of media likely to appeal to end-users.

A broad variety of media and formats were suggested, including: information seminars that included both technical specialists and growers experienced in dealing with the pest; support/extension personnel who could visit the farmer/ property owner with advice; various forms of media (TV, radio, websites, local newspapers, industry newsletters and magazines, council information mail-outs, etc.); as well as more detailed or scientific fact-sheets and best-practice guidelines. A primary concern, as indicated above, was that the messages conveyed through these various sources were consistent and thereby reinforced one another.

The quantitative survey sought to test the importance of knowledge and knowledge support (in the form of access to a technical or coordination support officer) with a much larger sample of growers and general public. Interestingly it found only a moderate level of influence on respondents' decisions to become involved in AWM (see also Section 3.4 in this chapter). Other factors were found to play a stronger role in decision-making, such as: perceptions of the level of threat 
posed by Queensland fruit fly, fairness in the implementation of AWM, costs and benefits of AWM, and social norms around what was the morally responsible thing to do (these psychosocial factors are described in detail below). Nevertheless, overall, it appears that the building of awareness and knowledge around Queensland fruit fly and its control through AWM, including the SIT, was considered a highly important component of any broader strategy to deal with it.

\subsection{Rules, Regulation and Enforcement}

A range of issues were identified in the qualitative studies relating to rules and regulations. Most issues concerned new or enhanced regulation considered necessary to improve success in implementing AWM to control Queensland fruit fly, while some related to current laws or regulations seen as likely to impede success. The main types of issues for which additional rules and regulations were seen as required have included:

- Ways to increase participation in AWM (e.g. mandating participation)

- Control of the pest in areas that were unmanaged or poorly controlled (e.g. removal of fruit-trees from abandoned orchards and backyards)

- Mandating specific control practices (e.g. orchard sanitation, backyard traps)

- Implementation of funding mechanisms to support AWM and SIT implementation (e.g. compulsory levies, accreditation schemes)

- Changes to market access protocols that would enable AWM and/or the SIT to be included.

A range of expectations were expressed around activities to limit the introduction of flies from elsewhere, even in areas where it had been declared endemic. In those regions where Queensland fruit fly had been declared endemic and the Fruit Fly Exclusion Zone (FFEZ) recently dismantled, there were calls for its reintroduction. In region $\mathrm{C}$, a regulated Queensland fruit fly-free area, calls were often made for the state government to ensure greater vigilance in inspections and/or fund additional routes where checkpoints should be implemented.

Two main types of currently existing rules and regulations were considered an impediment to AWM. Privacy laws preventing release of data mapping the distribution and hot spots of Queensland fruit fly in a region were viewed as impeding timely control responses by growers. Also considered an impediment were laws preventing growers or members of the public removing host vegetation on public lands, such as fruit trees on roadsides adjoining commercial properties.

A number of participants contended that currently existing laws and penalties needed to be better communicated or more rigorously monitored and enforced. In contrast, at a broader level some study participants contended that reliance on rules and regulations would be counter-productive and a more positive approach relying on communication, engagement and incentives would be more helpful. Participants also reported they would value pest density maps and location of hot spots if these were made available in a timely manner. They were seen of benefit for individual farm pest management and also to socially "pressure" others with pest problems to participate in management activities. 
Maintaining a Queensland fruit fly monitoring network of requisite density and ensuring timely reporting could, however, be expensive, raising questions of who should pay. Further, this "name and shame" approach was also considered potentially counter-productive by putting negligent growers off-side, further reducing collaborative behaviour.

\section{SOCIAL-PSYCHOLOGICAL BARRIERS AND FACILITATORS FOR AWM AND THE SIT}

An individual-level analysis of social psychological factors contributing to potential uptake of AWM practices and acceptance of the SIT was also conducted. This analysis provided important insight into how people living and working under institutional arrangements, discussed in Section 2, felt about AWM and the inclusion of the SIT in it. Furthermore, it was important to gain some baseline understanding of stakeholders' motivation to undertake AWM, possible factors affecting adherence to an AWM programme, and existing habitual on-farm behaviours for fruit fly management that would be affected by AWM. Overall, we found that being a farmer or a non-farmer was not an important driver of intentions to implement AWM or indeed accept the idea of AWM. Rather, it was the individual-level social psychological drivers that distinguished between different levels of acceptance of AWM and SIT integration, and intentions to implement AWM. Fig. 2 represents the varied individual factors that emerged from our research. Many of the highlighted social issues presented in this Section can be viewed as both barriers and facilitators to uptake of AWM, depending on the framing and messaging. A selection of dominant issues presented in Fig. 2 will be discussed here, and they will be described generally in terms of their influence on uptake of AWM and SIT.

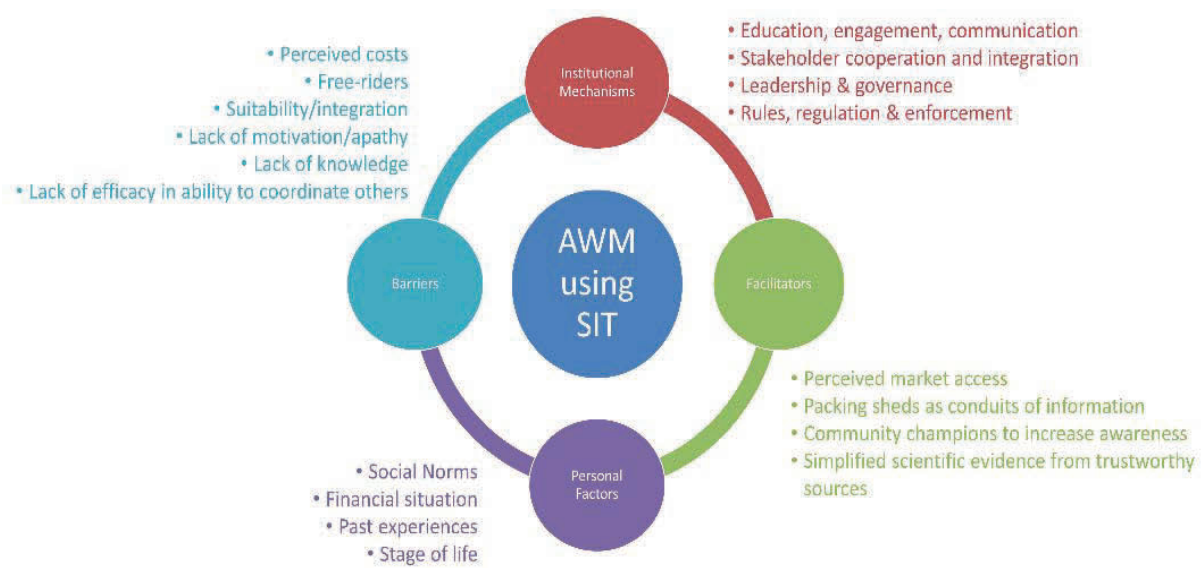

Figure 2. Key social factors influencing uptake of area-wide management (AWM) integrating the Sterile Insect Technique (SIT), framed as social barriers, facilitators, institutional mechanisms and personal factors. 


\subsection{Perceived Costs}

The primary consideration for farmers taking part in our studies was the perceived cost of AWM and the SIT. Specifically, people were interested in how much it would financially cost them to change current practices to conform to recommended AWM behaviours (e.g. orchard sanitation and hygiene, baiting and trapping). But, more so than AWM, people were keen to know more about the financial viability of SIT implementation and whether this type of technology could be used at the individual grower level in a cost-effective way. There was also a consistent view amongst participants that if relative costs of the SIT were perceived as prohibitive, then the largest barrier to widespread involvement in AWM would be this perception that the SIT was unaffordable.

Importantly, perceived costs of engaging in AWM did not only include monetary costs, but also personal costs such as time and effort. This was particularly evident for perceived costs associated with coordination efforts with neighbours, as part of AWM. Some participants believed that trying to coordinate growers across differing landscapes and production types would be difficult, if not impossible. The effort associated with organization, as well as the personal costs of potentially acting for the benefit of others, were thought to be difficult to overcome. This perception of response costs was found to be consistent across the three different regions, which did not significantly differ in their perceptions of anticipated costs associated with involvement in AWM.

Past psychological research on perceived costs has shown that those who acknowledge both costs and benefits to change achieved better outcomes than those acknowledging only benefits or costs, or few costs and benefits (McEvoy and Nathan 2007). Perceived costs can also be interpreted from the perspective of 'sunk costs', when one has already invested time and effort in developing on-farm habits, and financially invested in certain practices. A perception of costs within this framework could reflect how well a potential behaviour change (e.g. AWM) meets one's goals and aspirations, without too much of a conflict (Gifford 2011). Our results suggest that if AWM activities are perceived as requiring very little behavioural adjustment on an individual level, and/or the end goals meet individual needs, then the perception of costs will be low. A lowered perception of cost is related to a higher intention to engage in novel behaviours (Rogers 1983).

To cover the costs of an AWM with SIT programme, participants in our focus groups suggested a range of mechanisms:

- Funding by government (federal, state and/or local - an approach that tended to be favoured by growers)

- Various form of levies or hypothecated taxes (revenue from a specific tax earmarked for this purpose) on fruit production or fruit properties, urban households, or fruit sold to consumers, or

- A private user-pays model where fruit producers pay the entire cost.

Interestingly, while general public participants commonly suggested industry / growers and various levels of government should pay, they also appeared quite willing to pay something themselves, typically nominating local council taxes or levies imposed on retail fruit or urban households (see Nelson et al., this volume). 


\subsection{Compatibility of AWM with Existing On-farm Practices}

According to Rogers' (2003) diffusion of innovation theory, perceptions of compatibility with respect to an innovation will affect its adoption. The innovative practice tested in the present study, using diffusion of innovation principles as predictors, was AWM. Compatibility refers to how consistent the innovation is with the values, experiences and needs of the potential adopters. In the farming context, this also refers to how compatible AWM activities are with the existing on-farm practices.

Perceived compatibility of AWM with existing farming practices emerged as a driver of AWM uptake through our qualitative study. Through stakeholder interviews, we found that participants were primarily concerned about the coordination aspect of AWM. Specifically, the concern was that engaging in coordinated pest management efforts would be difficult because of different harvesting times and spraying times for different production enterprises within a region. Some participants perceived a significant risk if one group of farmers were spraying to reduce numbers in preparation for SIT, when another group was at a different stage in their harvesting cycle and could not do the same. The perception of incompatibility was primarily focused on collaboratively preparing a farming region for the introduction of the SIT. There were queries as to whether SIT application would or could be compatible for a whole region, or whether it was more suited to smaller scale releases, such as individual farm scale. Another compatibility issue raised was the logistical strain of setting up a systematic monitoring grid, as well as removing unwanted and fallen fruit on a commercial farm. The latter activity was perceived to be an immense task in itself and potentially incompatible with existing resource allocations.

Compatibility was also subsequently identified as an important driver for the prediction of public acceptance for AWM. Our quantitative research suggested that a key factor likely to influence community adoption of AWM practices was the expectation that AWM activities should complement rather than impede or complicate their current pest management behaviours. For farmers, this could mean capitalising on existing peer communication networks and sources of information to ensure coordination aspects of AWM are built into established social systems. It could also include more aggressive social cues, such as building up peer pressure at the community level to encourage farmers to remove infested and fallen fruit or sharing of monitoring data. For the general public, this may mean ensuring the messaging around AWM at the household level demonstrates an understanding of existing backyard habits, to mobilize the public so that hosts or fruit are effectively eliminated by all in a timely way along roads and in backyards. It could also mean acknowledging that the community is comprised of individuals with varying levels of interest and buy-in for AWM. Thus, compatibility would rest on AWM tasks that are presented as flexible, adaptable for those with different levels of intentions and willingness to participate, and new behaviours that are not perceived as an additional burden to household activities. 


\subsection{Free-riders and Fairness}

In a collaborative framework such as AWM, where the group benefits from shared action, there was an inherent concern for free-riders within the system. Certainly, much of the impetus driving social research in the context of an AWM scenario is a fear that a recalcitrant few could cause others to favour inaction in the name of fairness. The free-rider problem, at its core, is an economic problem describing those who benefit from goods, services or resources without contributing to payment (Baumol 1952). In the present study, we conceptualise the free-rider from a social psychological perspective. As such, 'free-riding' in this context comprises aspects of 'social loafing' (a psychological term), fairness, and conditional participation. Therefore, one is perceived to be free-riding if one is choosing to willingly disengage from a shared system but will still retain the resultant benefits of the shared system.

In psychological terms, the social loafing phenomenon describes a situation when an individual contributes less effort in a group setting, compared to when they work alone (Jackson and Harkins 1985; Karau and Williams 1993). In the context of AWM, that could be envisaged as a cluster of neighbouring growers working together to manage fruit flies. However, one grower in the middle of the region refuses to participate, whilst the others agree to implement coordinated activities. Consequently, the group coordinates and manages their pest management activities, while the lone grower in the middle refuses to implement any new practices because of different reasons. In such a scenario, the overall suppression of a pest will not be as strong compared to if all growers were participating in AWM. However, it is likely that the group will still work towards area-wide suppression even without the lone grower's involvement in the coordinated efforts of his/her neighbours. Thus, the lone grower is still likely to gain benefits from the collective efforts of the other growers, without exerting the same level of effort. This type of individualistic behaviour in the context of AWM can not only affect the success of managing a pest and coordination of efforts, but it can also be a deterrent for others to get involved. The importance of fairness in shared approaches such as AWM is paramount to its success.

The idea of perceived fairness emerged from our qualitative work as a key requirement for encouraging stakeholders to get involved in AWM. In particular, participants felt that growers who were harvesting non-dominant host crops, which would be less susceptible to Queensland fruit fly, would be more likely to question the fairness of AWM. These indirect beneficiaries of an AWM approach would likely need convincing of how AWM of the fruit fly was fair to people like themselves, who would directly benefit less from area-wide suppression of this pest. It was clear that, from a social justice perspective, AWM would need to appear advantageous to all involved, not just those growers who were growing Queensland fruit fly host plants. There was also a clear concern that growers of non-dominant host plants would be more likely to 'free-ride' the system, because of a perception that they may have less to gain.

While the qualitative research highlighted free-riding as a concern, our quantitative analysis helped to highlight the importance of fairness. Perceptions of fairness emerged as the single most dominant predictor of public acceptance for AWM. It was clear that acceptance for AWM amongst growers and members of the broader community were contingent upon how fair they believed AWM to be for 
everyone. In practical terms, this meant that no matter how simple or effective AWM might be, the strength of uptake may ultimately depend on all parties feeling as though AWM was a balanced approach.

\subsection{Knowledge}

Subjective or perceived knowledge (i.e. one's belief about personal knowledge on the subject matter), rather than actual knowledge, has been identified as a driver of protective action in the context of fear appeals (i.e. threats) in the psychological science literature (e.g. Martin et al. 2007; Nabi et al. 2008). Results from our present research found that the role of subjective knowledge could be examined more accurately by breaking it down into two: a) perceived knowledge of Queensland fruit fly, and b) knowledge of ways to manage this pest. Compared to members of the general public, growers had significantly greater knowledge of both Queensland fruit fly and technical methods of controlling Queensland fruit fly. However, subjective knowledge of Queensland fruit fly was moderate overall, and technical knowledge of control methods was low.

Qualitative results indicated that growers believed that they were already carrying out AWM. However, it was clear that growers had an inconsistent understanding of what AWM was, particularly the key role of coordination in AWM. Most growers interviewed believed that AWM was the management of one's whole property area, rather than a coordinated effort across potentially multiple properties within a given area. Predictive analyses further demonstrated that technical knowledge of Queensland fruit fly control was a direct predictor of intentions to implement AWM. Thus, those who felt they knew more about existing ways to control Queensland fruit fly were more likely to engage with AWM. Interestingly, however, subjective knowledge of Queensland fruit fly alone was not a predictor of intention to implement AWM.

An overall lack of knowledge of Queensland fruit fly amongst participants in our research may help to explain why perceived complexity of AWM was the secondmost dominant predictor of acceptance for AWM (after fairness). This suggests that while people were happy to support the use of AWM as a way to manage fruit flies, the topic was an area in which they held limited knowledge. Thus, people indicated that they would respond more positively to uptake of AWM if the information was simple and personal involvement was not too demanding.

Another motivational construct affected by knowledge and awareness is that of apathy. Stakeholders such as industry representatives, agronomists, and farmers, all felt that a significant problem in galvanising people within a region - whether farmers or the general public - was a perceived lack of understanding of the relevance of Queensland fruit fly control. Stakeholders felt there was a general lack of knowledge about how fruit fly contributed to horticultural and financial losses and a lack of market access. 


\subsection{Efficacy and Competence}

Self-efficacy, and the related concept of competence, have been found to be strong psychological drivers for behavioural action (Bandura 1977; Deci and Ryan 1995). The psychology literature describes self-efficacy as a belief in one's ability to accomplish a task. Self-efficacy can develop through observing modelled behaviour, feedback from others, and past experience or mastery of related behaviours. An important distinction to make, which will ultimately assist in the development of ways to promote self-efficacy on the ground, is that self-efficacy is not the same as selfconfidence. The latter refers to a broader sense of personal esteem and general likelihood of succeeding; in comparison, self-efficacy is more specifically tied to one's ability to perform a task. Self-efficacy is therefore more closely linked with a perception of competence, however, the concepts remain distinct in psychological theories (e.g. Rodgers et al. 2014). Ryan and Deci (2000) have described perception of competence as a fundamental driver of human motivation. Perceptions of competence reflect a self-awareness of individual capabilities and one's ability to control a situation. Past psychological research has demonstrated that engagement in protective behaviours (e.g. AWM of a pest) is significantly linked with how efficacious - or, competent - one feels with respect to the required behaviours.

In the present examination of public intentions to implement AWM, we measured self-efficacy towards carrying out behaviours recommended as part of an AWM approach. People were asked how easily they could carry out tasks such as removing unwanted fruit from trees and the ground, hanging out fruit fly traps, and coordinating with neighbours. We found that perceptions of self-efficacy related to these AWM activities were moderately high, but that self-efficacy was the strongest driver of intention to participate when taking into account other motivational factors such as knowledge of Queensland fruit fly and its management, severity and vulnerability of the problem, perception of response costs, and perceived effectiveness of AWM. People were most likely to engage in AWM if they felt they could do so successfully and competently.

This finding, on the ground, translates to a developmental need amongst potential participants of AWM. That is, fulfilling a desire for people to feel competent in engaging with AWM tasks through training and by providing opportunities for experiential learning and mastery. It is generally understood that within a community or regional group, there are people with diverse physical capabilities and interests, particularly related to a niche topic such as Queensland fruit fly, or even the broader notion of pests in the garden. What is less understood is, fundamentally, that the need to feel competent or efficacious when carrying out tasks is a driver for all human behaviour. Therefore, encouraging people to participate in a potentially unfamiliar activity will require addressing this universal need to feel capable.

\subsection{Community Champions}

A strong finding in our social research was that farmers and members of the general public were putting their trust in 'important others'. Important others are peers, family and friends whose opinions the individual trusts, or whose personal approval is 
important. Important others can also be members of the community - whether that be the farming community or influential members of the public. They are seen as local leaders, championing local causes and effecting change. Our results highlighted that these key individuals within a community were often seen as thought leaders, who kept the community's best interests at the forefront.

Amongst farmers, extension personnel such as agronomists were consistently identified as trusted sources of information. They were thought to be influential in positively changing on-farm practices and in providing practical support on the ground. In addition, the role of packing houses was also found to be valuable in conveying important information. Packing houses involved in packing and/or marketing fruit must also comply with export protocols. For domestic and international trade, packing houses undertake in-line inspections and are subject to audits. Therefore, the level of influence packing houses have on growers is substantial (Kruger 2016). Packing houses are familiar with compliance measures of good agricultural practices through international quality assurance schemes, such as Global G.A.P. or Freshcare, or the new Harmonised Australian Retailer Produce Scheme (HARPS 2017). Thus, packing houses could directly influence change in grower behaviours through their fruit acceptance requirements and standards.

For the general public, a community champion was represented as their local government council rather than a specific individual or type of individual. In Australia, local councils often lead community-level initiatives for the direct benefit of the community. It was clear from our qualitative research that the general public expected the local council to lead Queensland fruit fly area-wide control efforts in their town and provide guidance to residents on what activities to do. Our results, therefore, provided a good example of how local groups could also champion community causes.

Importantly, it was evident that community champions were a valuable conduit for information that might otherwise not get transmitted or distributed to some sections of society. For example, members of the target population, difficult to reach using traditional or mainstream communication strategies, may be more receptive to personal interactions for gaining information. The functionality of community champions in the context of AWM participation and SIT adoption was seen as important. Given their high level of trust within the community, it was possible that community champions could use their local influence to emphasise shared goals in the community, such as working towards a mutually beneficial outcome (e.g. economic growth or a cleaner environment). They could also serve as influential "messengers" for information or scientific evidence, and act as informal project managers for AWM. This could enable some sort of community-led 'enforcement' of AWM to ensure that actions to prevent/control fruit fly were being done on the ground.

Community champions can also provide a vital social support role for those experiencing difficulty in carrying out AWM or those who are unsure of the impacts of Queensland fruit fly, AWM or SIT adoption on their livelihoods or lifestyles. Typically, those championing causes at the scale of AWM are not passive participants in the social process (Norris et al. 2008; Conrad and Hilchey 2011). Rather, they have carried out their own investigations of available information and have a level of 
personal investment or buy-in for the ideals represented by the initiative. As a result, community champions are likely aware of local concerns and can support the change process through context-specific knowledge they have gained.

\subsection{Evidence}

The theme emerging from our data highlights the importance of contextual evidence of success using AWM and the SIT for Queensland fruit fly control. Both growers and members of the general public indicated that for wide-scale cooperation and buyin such as that required for AWM with an SIT component, they would need strong scientific evidence. This indicates the public values not only evidence of the science, but also an expectation that the public has access to evidence in a 'digestible' format (Blackstock et al. 2010; Garforth et al. 2013). This could take the form of public events conveying scientific results or creating science communication materials that highlight the science behind programmes such as AWM and the use of the SIT.

Experiential evidence was also an important factor for the acceptance of AWM as a pest control option, particularly for those living outside endemic areas. Growers stated they would be more likely to accept and participate in AWM if they could observe, first-hand, the presence of Queensland fruit fly, the damage it could do, the economic evidence that it was a problem, and that AWM was effective in reducing fly numbers. Similarly, industry stakeholder indicated that they would react more quickly on encouraging participation in AWM and the SIT use if they had evidence of what it means for their grower base, on-ground actions, and market access.

The SIT was also a technology that participants were unfamiliar with and while many growers and the general public believed it was an interesting idea in theory, they were clear that trusted and rigorous scientific evidence would be needed. Concerns remained around the notion that the SIT was safe, effective and worth the investment. It also appeared important for growers to see evidence that sterilisation works and that it is not a technique that is essentially still in trial stages. There was a fear that if the science is not correct, then growers may be used as unwilling guinea pigs on which to release untested sterile flies that may or may not be effective in controlling the wild fly population.

Notably, while scientific and experiential evidence was cited as being important, the source of such evidence would likely also be a significant factor (Twyman et al. 2008; Hernández-Jover et al. 2012). Therefore, any evidence communicated to the public would need to originate from trusted sources in order for evidence to serve as a facilitator for AWM and SIT adoption.

\section{THE ROLE OF THE SIT AS PART OF QUEENSLAND FRUIT FLY AWM}

Whilst the SIT has had a role in Queensland fruit fly outbreak responses for many years in south-eastern Australia (Reynolds et al. 2010), the SIT as a supplementary fruit fly management tool is a relatively novel concept for growers. Facts about the use of the SIT in managing Queensland fruit fly (e.g. increased efficacy under low pest prevalence conditions) are not well understood. However, the release of sterile male flies embedded into an AWM programme has proven to be highly successful 
overseas for other flies (Enkerlin et al. 2005; Pereira et al. 2013), as well as in Western Australia (Fisher et al. 1985; Fisher 1996).

The introduction of the SIT into AWM programmes for fruit fly suppression, containment or eradication is recommended for a number of reasons (Suckling et al. 2016). Permalloo et al. (2005) and Dyck et al. (2021) both consider the SIT as a sustainable pest management practice, and Hendrichs et al. (2007) consider it an effective environment-friendly tool with the ability to eradicate pests when used within an AWM programme. The SIT is considered pest-specific and compatible with existing tools (Vargas et al. 2008; Dyck et al. 2021; Enkerlin 2021).

McInnis et al. (2005) and Vargas et al. (2008) agree that male-only releases of the SIT is more effective than a bisexual line when using sterile flies; both for the mating ratio that is imposed, but also for the reduced risk of fruit damage by ovipositing female flies. McInnis et al. (2005) and Meats et al. (2003) suggest the sterile to wild fly ratio in trap catches can be effective in assessing the SIT, and McInnis et al. (2005) further posit that when sterile flies released are competitive, a sterile to wild fly ratio at 10:1 (in traps) shows effectiveness. This highlights the importance of a trapping network to monitor the distribution and abundance of both wild and sterile flies and the success of SIT programmes.

\subsection{Institutional Considerations}

The scope, scale and method of release of sterile flies will affect sterile fly quality and have implications for the management and governance arrangements for a SIT programme (Vreysen et al. 2007b). At the time of this study, details of these for the three study regions were limited, and a range of possibilities were canvassed in qualitative forums. Suggestions included:

- Small-scale on-farm release by individual growers coordinating at some level with other growers in an AWM approach

- AWM grower groups working together using farm vehicles or aircraft across a larger growing area

- Urban only releases to suppress pest populations in towns, or

- Broad-scale coverage of both commercial and urban areas, typically using aircraft. Each approach is likely to require a different management arrangement and governance consideration.

Bottom-up approaches that rely on individual growers or AWM grower groups acting independently from a central authority, for example purchasing the sterile flies from a local supplier - much as they currently do for other Queensland fruit fly control techniques (bait spray, traps, chemical sprays, nets) - would, in principle, involve limited supervision. However, much experience with AWM with SIT programmes has shown they tend to be management intensive, requiring professionals dedicated to SIT implementation, and some form of central coordinating body (Vreysen et al. 2007b), such as a regional Queensland fruit fly control coordinating committee. Tasks include gathering information to monitor SIT releases in the region and compare sterile and wild fly populations to assess programme effectiveness. 
Broader-scale AWM and releases managed by a central authority, such as a regional coordinating committee, will require an even higher level of management. Vreysen et al. (2007b) pose seven questions that need to be addressed by managers of any AWM with SIT scheme:

1. Is the programme management structure that includes full-time professionals sufficiently flexible and independent from the regional or federal government bureaucracy?

2. Is there sufficient commitment by key stakeholders so that there is a high degree of continuity in implementation of all essential programme components?

3. Are funding mechanisms established and available resources, manpower and institutional capacity sufficient in magnitude and quality to assure effective operations and sustainability of the programme?

4. Does the programme have the support of all stakeholders and firm commitments from those who must bear costs or conduct relevant operations?

5. Are essential high-quality data being collected and properly analysed in a timely manner to enable the programme management to provide feedback essential for corrective action by all key programme personnel?

6. Is the public awareness and public education programme of sufficient quality to help shape attitudes and behaviours in support of programme success?

7. Is the programme benefiting from timely and independent reviews?

At a broader level, SIT integration raised questions among participants in this study about the need for modification of market access protocols when including the SIT as a control strategy. This was perceived as a long and arduous process, as each market access protocol is specific to each of the many different countries of destination, and often requires unique specifications for each type of fruit and vegetable. Any protocol changes must be centrally managed through the federal department of agriculture in consultation with state and territory governments, whose own legislation may need modifying to accommodate changes. Furthermore, the federal department of agriculture is typically negotiating many other pest and biosecurity priorities with each country. For these reasons it was felt by some that the SIT may be of limited value in opening up new market access, at least in the short term.

Participants in our qualitative studies also raised questions on the implication of some aspects of SIT application that may present impediments to their acceptance by protocol markets. For example, increased fly counts in traps of monitoring grids, due to the trapping of sterile flies, would require willingness of protocol markets to accept assurances about distinctions with wild fly counts and count veracity. However, this perception may prove to be an unfounded fear, as many major horticultural exporting countries use the SIT extensively and presumably have found ways of addressing the issue (e.g. Enkerlin et al. 2015; Enkerlin 2021).

\subsection{Social Acceptance}

The evidence from our programme of social research has indicated a high level of social acceptance for the notion of adopting the SIT as part of AWM. Growers consistently perceived the SIT as another potential tool in their toolbox for 
suppressing or eradicating Queensland fruit fly. There was evidence that some growers were cautious in their assessment of the SIT, moderating personal expectations of the success of SIT application. These individuals cited that while the SIT sounded promising, their history with fruit fly showed them that there was no one answer to successfully controlling the pest. They correctly felt that the SIT could only be useful in conjunction with an AWM approach or other stringent control activities. Most growers felt that, other than cost, there would be no real barriers to the adoption of the SIT and pilot trials should begin. Associated with this was a sense of frustration amongst some growers, who believed that authorities 'holding on' to the technology, without implementing it in the field, left growers waiting. Thus, it seems that there was a complex association with the idea of SIT application amongst growers. On the one hand, growers were not keen to be trial subjects for a technology that according to them wasn't ready (and lacked the scientific evidence for the efficacy that they demanded). Yet, on the other hand, they were keen to see SIT implemented quickly, although it was generally not understood by them that an effective AWM system needs first to be in place.

An interesting result that emerged from our stakeholder interviews was a perception that the general public would be very accepting of the SIT. This was primarily driven by a belief that townspeople would not be unduly affected by the release of sterile flies, nor would they be involved in organising SIT releases. Therefore, there was nothing that townspeople could object to. The qualitative data were subsequently supported by our quantitative results, which indicated a high level of general acceptance for SIT integration amongst both growers and the general public. While general acceptance for SIT application was high overall, there were many questions about the sterile fly itself and, in particular, its sterility. However, these questions seemed to be driven by curiosity rather than a desire to question the legitimacy of the sterilisation technique.

Finally, when stakeholders and growers were asked about implementing a SIT programme, many respondents felt as though there was no real need for consultation because it was a simple concept to explain. Growers in particular felt that the SIT would be readily accepted amongst their peers. This is because participants believed that growers were so desperate for a long-term solution to Queensland fruit fly that anything was better than nothing at this point. The general public, however, did indicate that while general perceptions of the SIT were positive, they had many questions. Therefore, it was clear that general public acceptance for SIT application was conditional upon having the appropriate level of knowledge to make an informed decision.

\section{CONCLUSIONS}

This social science research into acceptance of AWM with a SIT component was undertaken to measure drivers and barriers to uptake of both a management style in the form of AWM, as well as a novel technology such as the SIT for Queensland fruit fly control. Most growers held an inconsistent understanding of AWM and were not aware of the significant coordination of effort required for true AWM. In fact, most growers felt that because they were managing pests on their own farms, they were 
effectively performing AWM. Overall, growers were willing to be involved in AWM as long as they felt competent to do so. Growers perceived a severe threat, and involvement in AWM was fair to everyone involved. Feelings of over-contributing with minimal benefits and a lack of cooperation, along with perceptions of financial costs, were seen to be potential barriers of uptake. Therefore, the crucial 'coordination' aspect of AWM was considered a significant barrier to overcome, depending on the scale of coordination required.

Aside from the involvement of growers, AWM in the regions examined within this study would also likely involve the participation of townspeople. Indeed, the survey results showed the general public were accepting AWM, as long as they were guided in the right direction and area-wide activities were simple. This perception of high general public interest was related to a belief that AWM in towns would require little investment at the individual level, such as time and cost. For example, simple backyard fruit fly traps could be subsidised by local government councils such that individual property owners would only contribute a nominal amount. In reality, this perception of a low level of engagement from householders may not be realistic and involvement in AWM may require greater effort than anticipated.

Funding the costs of implementing AWM with the SIT, whether applied only in urban areas or additionally in surrounding commercial fruit growing areas, will also require serious thought. Funding into the future will be needed, in particular for education efforts, technical and coordination personnel, subsidisation of monitoring grids and traps in urban areas and, in the case of SIT, for the supply and application of sterile flies. Fortunately, our research results indicated participants were willing to entertain a range of potential funding mechanisms, including levies on urban households.

Finally, the take-home message from our social research was that growers and the general public alike still need considerable education to have a realistic understanding of what AWM and SIT implementation entails. Fortunately, both participant groups were influenced by the behaviours of important others (i.e. social norms). If certain attitudes and behaviours were modelled for them, then growers and non-growers were likely to be more receptive to accepting AWM and SIT use, and also more likely to participate in the pest management approach. These findings were consistent across regions and social contexts, suggesting that the influence of social factors has the potential to contribute to an underlying understanding of drivers and barriers for AWM and SIT uptake.

\section{ACKNOWLEDGMENTS}

The Adaptive Area Wide Management of Queensland fruit fly using the SIT project is being delivered by Hort Innovation in partnership with CSIRO and is supported by funding from the Australian Government Department of Agriculture \& Water Resources as part of its Rural R\&D for Profit programme. Further partners include Agriculture Victoria, NSW DPI, PIRSA, QUT, SARDI, Wine Australia and BioFly. We also acknowledge the valuable contribution of reviewers in improving the quality of this manuscript. 


\section{REFERENCES}

Abdalla, A., N. Millist, B. Buetre, and B. Bowen. 2012. Benefit-cost analysis of the National Fruit Fly Strategy Action Plan. ABARES report to client prepared for Plant Health Australia, December 2012. Canberra, Australia.

Agriculture Victoria. 2017. Queensland fruit fly: Declared outbreak zones. Retrieved May 22, 2018.

Bandura, A. 1977. Self-efficacy: Toward a unifying theory of behavioural change. Psychological Review 84: $191-215$

Barclay, H. J., R. Matlock, S. Gilchrist, D. M. Suckling, J. Reyes, W. R. Enkerlin, and M. J. B. Vreysen. 2011. A conceptual model for assessing the minimum size area for an Area-Wide Integrated Pest Management program. International Journal of Agronomy: 409328.

Baumol, W. J. 1952. The transactions demand for cash: An inventory theoretic approach. Oxford Journals 66: $545-556$.

Blackstock, K. L., J. Ingram, R. Burton, K. M. Brown, and B. Slee. 2010. Understanding and influencing behaviour change by farmers to improve water quality. Science of the Total Environment 408: 5631-5638.

Chandler, L. D., M. M. Ellsbury, and W. D. Woodson. 1999. Area-wide management zones for insects. PPI Site-Specific Management Guidelines SSMG-19. South Dakota State University, Brookings, South Dakota, USA.

(CGA) Cherry Growers Association. 2015. Cherry export manual and biosecurity management program, Cherry Growers, Australia.

Clarke, A. R., K. James, J. Luck, M. Robinson, P. Taylor, and D. Barbour. 2014. The National Fruit Fly Research, Development \& Extension Plan. Plant Biosecurity Cooperative Research Centre. Department of Industry and Science, Canberra, Australia.

Conrad, C. C., and K. G. Hilchey. 2011. A review of citizen science and community-based environmental monitoring: Issues and opportunities. Environmental Monitoring and Assessment 176: 273-291.

Deci, E. L., and R. M. Ryan. 1995. Human autonomy: The basis for true self-esteem, pp. 31-49. In M Kernis (ed.), Efficacy, agency, and self-esteem. Plenum, New York, USA.

Dominiak, B.C., and D. Daniels. 2012. Review of the past and present distribution of Mediterranean fruit fly (Ceratitis capitata Wiedemann) and Queensland fruit fly (Bactrocera tryoni Froggatt) in Australia. Australian Journal of Entomology 51: 104-115.

Dominiak, B. C., and J. H. Ekman. 2013. The rise and demise of control options for fruit fly in Australia. Crop Protection 51: 57-67.

Dominiak, B. C., and R. Mapson. 2017. Revised distribution of Bactrocera tryoni in eastern Australia and effect on possible incursions of Mediterranean fruit fly: Development of Australia's eastern trading block. Journal of Economic Entomology 110: 2459-2465.

Dominiak, B. C., B. Wiseman, C. Anderson, B. Walsh, M. McMahon, and R. Duthie. 2015. Definition of and management for areas of low pest prevalence for Queensland fruit fly Bactrocera tryoni Froggatt. Crop Protection 72: 41-46.

Dyck, V. A., J. Hendrichs, and A. S. Robinson (eds.). 2021. Sterile Insect Technique - Principles and practice in Area-Wide Integrated Pest Management. Second Edition. CRC Press, Boca Raton, Florida, USA. 1200 pp.

Enkerlin, W. R. 2021. Impact of fruit fly control programmes using the Sterile Insect Technique, pp. 9791006. In V. A. Dyck, J. Hendrichs, and A. S. Robinson (eds.), Sterile Insect Technique - Principles and practice in Area-Wide Integrated Pest Management. Second Edition. CRC Press, Boca Raton, Florida, USA.

Enkerlin, W., J. M. Gutiérrez-Ruelas, A. V. Cortes, E. C. Roldan, D. Midgarden, E. Lira, J. L. Z. López, J. Hendrichs, P. Liedo, and F. J. T. Arriaga. 2015. Area freedom in Mexico from Mediterranean fruit fly (Diptera: Tephritidae): A review of over 30 years of a successful containment program using an integrated area-wide SIT approach. Florida Entomologist 98: 665-681.

Ekman, J. 2015. Pests, diseases and disorders of sweet corn: A field identification guide. Applied Horticultural Research. Horticulture Innovation Australia Limited. Eveleigh, New South Wales, Australia.

Fisher, K. T., A. R. Hill, and A. N. Sproul. 1985. Eradication of Ceratitis capitata (Wiedemann) (Diptera: Tephritidae) in Carnarvon, Western Australia. Australian Journal of Entomology 24(3): 207-208.

Fisher, K. T. 1996. Queensland fruit fly (Bactrocera tryoni): Eradication from Western Australia, pp. 535 541. In B. A. McPheron, and G. J. Steck (eds.), Fruit fly pests, a world assessment of their biology and management. St. Lucie Press, Delray Beach, Florida, USA. 
Florec, V., R. J. Sadler, B. White, and B. C. Dominiak. 2013. Choosing the battles: The economics of area wide pest management for Queensland fruit fly. Food Policy 38: 203-213.

(FAO) Food and Agriculture Organization of the United Nations. 2016. Establishment of pest free areas for fruit flies (Tephritidae). International Standard for Phytosanitary Measures (ISPM) No. 26. International Plant Protection Convention. Rome, Italy.

Garforth, C. J., A. P. Bailey, and R. B. Tranter. 2013. Farmers' attitudes to disease risk management in England: A comparative analysis of sheep and pig farmers. Preventive Veterinary Medicine 110: 456466.

Giddens, A. 1984. The constitution of society: Outline of the theory of structuration. Polity Press. Cambridge, UK.

Gifford, R. 2011. The dragons of inaction: Psychological barriers that limit climate change mitigation and adaptation. American Psychology 66: 290-302.

(HARPS) Harmonised Australian Retailer Produce Scheme. 2017. Horticulture Innovation Australia.

Hendrichs, J., P. Kenmore, A. S. Robinson, and M. J. B. Vreysen. 2007. Area-Wide Integrated Pest Management (AW-IPM): Principals, practice and prospects, pp. 3-33. In M. J. B. Vreysen, A. S. Robinson, and J. Hendrichs (eds.), Area-wide control of insect pests: From research to field implementation. Springer, Dordrecht, The Netherlands.

Hernández-Jover, M., M. Taylor, P. Holyoake, and N. Dhand. 2012. Pig producers' perceptions of the influenza pandemic H1N1/09 outbreak and its effect on their biosecurity practices in Australia. Preventive Veterinary Medicine 106: 284-294.

Hodgson, G. M. 2006. What are institutions? Journal of Economic Issues 40: 1-25.

Hodgson, G. M. 2007. Institutions and individuals: Interaction and evolution. Organization Studies 28: 95-116.

Jackson, J. M., and S. G. Harkins. 1985. Equity in effort: An explanation of the social loafing effect. Journal of Personality and Social Psychology 49: 1199-1206.

Karau, S. J., and K. D. Williams. 1993. Social loafing: A meta-analytic review and theoretical integration. Interpersonal Relations and Group Processes 65: 681-706.

Kruger, H. 2016. Designing local institutions for cooperative pest management to underpin market access: The case of industry-driven fruit fly area-wide management. International Journal of the Commons 10: 176-199.

Kruger, H. 2017a. Creating an enabling environment for industry-driven pest suppression: The case of suppressing Queensland fruit fly through area-wide management. Agricultural Systems 156: 139-148.

Kruger, H. 2017b. Helping local industries help themselves in a multi-level biosecurity world - Dealing with the impact of horticultural pests in the trade arena. NJAS - Wageningen Journal of Life Sciences 83: $1-11$.

Lloyd, A. C., E. L. Hamacek, R. A. Kopittke, T. Peek, P. M. Wyatt, C. J. Neale, M. Eelkema, and H. Gu. 2010. Area-wide management of fruit flies (Diptera: Tephritidae) in the Central Burnett district of Queensland, Australia. Crop Protection 29: 462-469.

Mankad, A. 2016. Psychological influences on biosecurity control and farmer decision-making. A review. Agronomy for Sustainable Development 36: 40.

Mankad, A., B. Loechel, and P. F. Measham. 2017. Psychosocial barriers and facilitators for area-wide management of fruit fly in southeastern Australia. Agronomy for Sustainable Development 37: 67.

Martin, I. M., H. Bender, and C. Raish. 2007. What motivates individuals to protect themselves from risks: The case of wildland fires. Risk Analysis 27: 887-900.

McEvoy, P. M., and P. Nathan. 2007. Perceived costs and benefits of behavioral change: Reconsidering the value of ambivalence for psychotherapy outcomes. Journal of Clinical Psychology 63: 1217-1229.

McInnis, D., R. F. L. Mau, S. Tam, R. Lim, J. Komatsu, L. Leblanc, D. Muromoto, R. Kurashima, and C. Albrecht. 2005. Development and field release of genetic sexing strains of the melon fly, Bactrocera cucurbitae, and Oriental fruit fly, B. dorsalis, in Hawaii. Extended synopses of the FAO/IAEA International Conference on Area-Wide Control of Insect Pests: Integrating the sterile insect and related nuclear and other techniques, Vienna, Austria.

Meats, A. 1981. The bioclimatic potential of the Queensland fruit fly, Dacus tryoni, in Australia. Proceedings of the Ecological Society of Australia 11: 151-161.

Meats, A. W., R. Duthie, A. D. Clift, and B. C. Dominiak. 2003. Trials on variants of the Sterile Insect Technique (SIT) for suppression of populations of the Queensland fruit fly in small towns neighbouring a quarantine (exclusion) zone. Australian Journal of Experimental Agriculture 43: 389-395.

Nabi, R. L., D. Roskos-Ewoldsen, and F. D. Carpentier. 2008. Subjective knowledge and fear appeal effectiveness: Implications for message design. Health Communications 23: 191-201. 
Norris, F. H., S. P. Stevens, B. Pfefferbaum, K. F. Wyche, and R. L. Pfefferbaum. 2008. Community resilience as a metaphor, theory, set of capacities, and strategy for disaster readiness. American Journal of Community Psychology 41: 127-150.

North, D. C. 1990. Institutions, institutional change and economic performance. Cambridge University Press, Cambridge, UK.

Ogaugwu, C. E. 2014. Towards area-wide control of Bactrocera invadens: Prospects of the Sterile Insect Technique and molecular entomology. Pest Management Science 70: 524-527.

Pereira, R., B. Yuval, P. Liedo, P. E. A. Teal, T. E. Shelly, D. O. McInnis, and J. Hendrichs. 2013. Improving sterile male performance in support of programmes integrating the Sterile Insect Technique against fruit flies. Journal of Applied Entomology 137: 178-190.

Permalloo, S., S. I. Seewooruthun, P. Sookar, M. Alleck, and B. Gungah. 2005. Area-wide fruit fly control in Mauritius. In Extended synopses of the FAO/IAEA International Conference on Area-Wide Control of Insect Pests: Integrating the sterile insect and related nuclear and other techniques, Vienna, Austria.

Reynolds, O. L., B. C. Dominiak, and B. A. Orchard. 2010. Pupal release of the Queensland fruit fly, Bactrocera tryoni (Froggatt) (Diptera: Tephritidae), in the Sterile Insect Technique: Seasonal variation in eclosion and flight. Australian Journal of Entomology 49: 150-159.

Reynolds, O. L., and B. A. Orchard. 2015. Roving and stationary release of adult sterile Queensland fruit fly, Bactrocera tryoni (Froggatt) (Diptera, Tephritidae). Crop Protection 76: 24-32.

Rodgers, W. M., D. Markland, A. M. Selzler, T. C. Murray, and P. M. Wilson. 2014. Distinguishing perceived competence and self-efficacy: An example from exercise. Research Quarterly for Exercise and Sport 85: 527-539.

Rogers, E. M. 2003. Diffusion of Innovations Theory. New York Free Press 5th ed. New York, USA.

Rogers, R. W. 1983. Cognitive and physiological processes in fear appeals and attitude change: A revised theory of protection motivation, pp. 153-176. In J. Cacioppo, and R. Petty (eds.), Social psychophysiology. Guilford Press, New York, USA.

Ryan, R. M., and E. L. Deci. 2000. Self-determination theory and the facilitation of intrinsic motivation, social development, and well-being. American Psychology 55: 68-78.

Stringer, L. D., J. M. Kean, J. R. Beggs, and D. M. Suckling. 2017. Management and eradication options for Queensland fruit fly. Population Ecology 59: 259-273.

Suckling, D. M., L. D. Stringer, A. E. A. Stephens, B. Woods, D. G. Williams, G. Baker, and A. M. El-Sayed. 2014. From Integrated Pest Management to integrated pest eradication: Technologies and future needs. Pest Management Science 70: 179-189.

Suckling, D. M., J. M. Kean, L. D. Stringer, C. Caceres-Barrios, J. Hendrichs, J. Reyes-Flores, and B. C. Dominiak. 2016. Eradication of tephritid fruit fly pest populations: Outcomes and prospects. Pest Management Science 72: 456-465.

Sutherst, R. W., B. S. Collyer, and T. Yonow. 2000. The vulnerability of Australian horticulture to the Queensland fruit fly, Bactrocera (Dacus) tryoni, under climate change. Australian Journal of Agricultural Research 51: 467-480.

Twyman, M., N. Harvey, and C. Harries. 2008. Trust in motives, trust in competence: Separate factors determining the effectiveness of risk communication. Judgment and Decision Making 3: 111-120.

Vander Meer, R. K., R. M. Pereira, S. D. Porter, S. M. Valles, and D. H. Oi. 2007. Area-wide suppression of invasive fire ant Solenopsis spp. populations, pp. 487-496. In M. J. B. Vreysen, A. S. Robinson, and J. Hendrichs (eds.), Area-wide control of insect pests: From research to field implementation. Springer, Dordrecht, The Netherlands.

Vargas, R. I., R. F. L. Mau, E. B. Jang, R. Faust, and L. Wong. 2008. The Hawaii fruit fly area-wide pest management programme. Publications from USDA-Agricultural Research Service/University of Nebraska Lincoln Faculty Paper 656.

Vargas, R., M. Detto, D. D. Baldocchi, and M. F. Allen. 2010. Multiscale analysis of temporal variability of soil CO2 production as influenced by weather and vegetation. Global Change Biol. 16: 1589-1605.

Vreysen, M. J. B., A. S. Robinson, and J. Hendrichs (eds.). 2007a. Area-wide control of insect pests: From research to field implementation. Springer, Dordrecht, The Netherlands.

Vreysen, M. J. B., J. Gerardo-Abaya, and J. P. Cayol. 2007b. Lessons from Area-Wide Integrated Pest Management (AW-IPM) programmes with an SIT component: An FAO/IAEA perspective, pp. 723 744. In M. J. B. Vreysen, A. S. Robinson, and J. Hendrichs (eds.), Area-wide control of insect pests: From research to field implementation. Springer, Dordrecht, The Netherlands.

Yu, R., and P. Leung. 2011. Estimating the economic benefits of area-wide pest management: An extended framework with transport cost. Annals of Regional Science 46: 455-468. 